\title{
O papel das bibliotecas durante a pandemia da Covid-19: em busca da emancipação humana
}

\author{
The paper of libraries during the Covid-19 pandemic: in search of human emancipation
}

\section{Letícia Rodrigues Santos}

Mestranda em Educação Profissional e Tecnológica Instituto Federal de Educação, Ciência e Tecnologia Goiano

leticia.santos@ifgoiano.edu.br

Morgana Bruno Henrique Guimarães

Mestranda em Educação Profissional e Tecnológica Instituto Federal de Educação, Ciência e Tecnologia Goiano

morgana.guimaraes@ifgoiano.edu.br

Leonardo Henrique Silva

Mestranda em Educação Profissional e Tecnológica Instituto Federal de Educação, Ciência e Tecnologia Goiano

leonardo.silva@ifgoiano.edu.br

Juliana Cristina da Costa Fernandes

Doutora em Educação

Instituto Federal de Educação, Ciência e Tecnologia Goiano

juliana.fernandes@ifgoiano.edu.br
Elisângela Ladeira de Moura Andrade

Mestranda em Educação Profissional e Tecnológica Instituto Federal de Educação, Ciência e Tecnologia Goiano

elisladeirama@gmail.com

Pedro Henrique Isaías

Graduando em Ciência da Computação Instituto Federal de Educação, Ciência e Tecnologia Goiano

pedrohisaias@gmail.com

Emmanuela Ferreira de Lima Doutora em Química

Instituto Federal de Educação, Ciência e Tecnologia Goiano

emmanuela.lima@ifgoiano.edu.br

Fernando Barbosa Matos

Doutor em Engenharia Elétrica

Instituto Federal de Educação, Ciência e Tecnologia Goiano

fernando.matos@ifgoiano.edu.br

\section{Resumo}

As bibliotecas possuem papel fundamental perante a sociedade. Dentre suas funções, está a colaboração para a formação de cidadãos conscientes e críticos, em face das informações que têm acesso. Com o intuito de atender essa função, o Sistema Integrado de Bibliotecas do Instituto Federal Goiano promoveu um evento em comemoração à Semana Nacional do Livro e da Biblioteca, totalmente em ambiente virtual, devido à pandemia da Covid-19. Dentre as diversas ações promovidas, realizou-se uma mesa redonda na plataforma Youtube, com o objetivo de discutir o tema fake news e inteligência artificial, no contexto pandêmico, e suas implicações na sociedade. A pesquisa apresenta o conceito de Bibliotecas dos Institutos Federais, sob o prisma da educação profissional e tecnológica, e evidencia o evento supracitado, com foco na formação de estudantes emancipados. Elaborou-se uma pesquisa de abordagem qualitativa, descritiva-exploratória, baseada em revisão de literatura, para a fundamentação teórica sobre bibliotecas, fake news e inteligência artificial, e também sobre emancipação humana. Concluiu-se que eventos de promoção à emancipação dos estudantes são muito relevantes para a formação de cidadãos conscientes, críticos e reflexivos, que podem agir com autonomia e ser protagonistas na sociedade a qual pertencem.

Palavras-Chave: Bibliotecas. Fake news. Inteligência artificial. Educação profissional e tecnológica. Instituto Federal Goiano. 


\begin{abstract}
Libraries have a fundamental paper in society. Among its functions, it is the collaboration for the formation of conscious and critical citizens, in the face of the information they have access to. In order to fulfill this function, the Integrated System of Libraries of the Goiano Federal Institute promoted an event in celebration of the National Book and Library Week, entirely in a virtual environment, due to the Covid-19 pandemic. Among the various actions promoted, a round table was held on the Youtube platform, with the objective of discussing the topic fake news and artificial intelligence, in the pandemic context and its implications for society. The research presents the concept of Libraries of the Federal Institutes, under the prism of the professional and technological education, and to highlight the aforementioned event, focusing on the formation of emancipated students. A qualitative, descriptive-exploratory research, based on a literature review, was developed for the theoretical foundation on libraries, fake news and artificial intelligence, and also on human emancipation. It was concluded that events to promote the emancipation of students are very relevant for the formation of conscious, critical and reflective citizens, who can act with autonomy and be protagonists in the society to which they belong.
\end{abstract}

\title{
Keywords
}

Libraries. Fake news. Artificial intelligence. Professional and technological education. Goiano Federal Institute.

\section{INTRODUÇÃO}

As bibliotecas dos Institutos Federais de Educação, Ciência e Tecnologia (IFs) têm a missão de amparar suas instituições mantenedoras, no que diz respeito à informação, e dar subsídios para o processo de ensino e aprendizagem dos seus estudantes, oferecendo produtos e serviços que viabilizem a formação humana integral e emancipadora, recomendada pela Educação Profissional e Tecnológica (EPT). Essa formação tem o intuito de proporcionar o desenvolvimento multidimensional dos estudantes, não havendo indissociabilidade entre trabalho, ciência, tecnologia e cultura, proporcionando uma visão histórica e cultural dos indivíduos, em busca de sua emancipação.

Para que tal formação se efetive, as bibliotecas dos IFs elaboram vários projetos e ações voltados aos seus usuários, com o intuito de fomentar o desenvolvimento humano integral. No entanto, em 2020, o mundo foi acometido por uma pandemia causada pelo novo coronavírus (SARS-CoV-2), ocasionado pela doença Covid-19, que apresenta um espectro clínico variando de infeç̧ões assintomáticas a quadros graves na população contaminada.

Nesse contexto pandêmico, o distanciamento social tornou-se necessário, para que o vírus não se propagasse tão rapidamente e sobrecarregasse os sistemas de saúde. Por esse motivo, os eventos presenciais não eram recomendados pela Organização Mundial da Saúde (OMS). Ponderando a situação descrita, o Sistema Integrado de Bibliotecas (SIBi) do Instituto Federal Goiano (IF Goiano) idealizou a Semana Nacional do Livro e da Biblioteca (SNBL), totalmente em ambiente virtual, aspirando às condições sanitárias exigidas no momento.

A SNBL é realizada anualmente pelo SIBi/IF Goiano e está em sua sexta edição, trazendo em sua programação temas relevantes a serem discutidos pela comunidade acadêmica, normalmente associadas ao tema da Semana Nacional de Ciência e Tecnologia (SNCT). Na edição de 2020, o tópico selecionado foi: "Fake News: o lado incógnito da Inteligência Artificial", aliado ao tema da SNCT: "Inteligência Artificial: a nova fronteira da ciência brasileira", por considerar que informações falsas podem ser prejudiciais ao processo emancipatório dos estudantes, bem como à toda a sociedade. 
O presente trabalho tem como objetivos: 1) discutir o tema fake news e inteligência artificial no contexto pandêmico, pois o cenário informacional foi expandido com a Covid-19, porém, nem todas as informações compartilhadas são verídicas, muitas vezes elas trazem conteúdos com o intuito de confundir ou enganar a população sobre a realidade que estamos vivenciando; 2 ) apresentar o conceito de Bibliotecas dos Institutos Federais, sob o prisma da educação profissional e tecnológica, relatando o projeto: "VI Semana Nacional do Livro e da Biblioteca", em ambiente virtual, para o desenvolvimento do processo emancipatório dos estudantes.

Concluiu-se que as atividades elaboradas pelas bibliotecas são importantes para levantar discussões e trazer conteúdos relevantes, os quais estão acontecendo na sociedade, evidenciando o papel social das bibliotecas na busca da emancipação humana.

\section{PROCEDIMENTOS METODOLÓGICOS}

O presente trabalho tem como base a live "Fake News - o lado incógnito da Inteligência Artificial", realizada em 2020 durante a sexta edição da Semana Nacional do Livro e da Biblioteca, sendo uma iniciativa do SIBi do Instituto Federal Goiano (IF Goiano). O evento realizou-se de forma on-line e gratuito. De acordo com Pereira, Shitsuka, Parreira \& Shitsuka (2018, p. 94-95),

[...] quando se escreve um artigo científico, está se relatando resultados de alguma descoberta, experiência realizada, descrevendo algum caso, relatando alguma experiência vivida, descrevendo algum fenômeno ocorrido ou ainda fazendo uma revisão bibliográfica em relação a algum tema.

Para a realização deste trabalho, desenvolveu-se uma pesquisa de abordagem qualitativa, de caráter descritivo-exploratório, visto que, sob a ótica de Matias-Pereira (2016, p.87), essa abordagem "parte do entendimento de que existe uma relação dinâmica entre o mundo real e o sujeito, isto é, um vínculo indissociável entre o mundo objetivo e a subjetividade do sujeito".

A fundamentação teórica baseou-se na revisão de literatura que, segundo o mesmo autor, "consiste em referenciar os estudos anteriormente publicados, buscando posicionarse sobre a evolução do assunto", para elencar os conceitos sobre fake news e inteligência artificial, além de concepções sobre as bibliotecas, EPT e emancipação.

\section{EMANCIPAÇÃO DOS ESTUDANTES DA EDUCAÇÃO PROFISSIONAL E TECNOLÓGICA}

Os IFs propõem uma formação que contempla a preparação para a vida e para o trabalho. Trabalho, neste caso, definido como uma categoria ontológica, inerente à espécie humana, na busca pela sua existência (MOURA, 2007). Porém, não se pode considerar que a proposta é ofertar dois tipos de formações diferentes, não pode haver conflito entre os objetivos. Pelo contrário, há que se pensar na integração deles, havendo uma formação única, que contemple o ensino propedêutico e a preparação para o trabalho. A formação integrada, conforme Ciavatta (2005, p. 85),

[...] sugere superar o ser humano dividido historicamente pela divisão social do trabalho entre a ação de executar e a ação de pensar, dirigir ou planejar. Trata-se 


\begin{abstract}
de superar a redução da preparação para o trabalho ao seu aspecto operacional, simplificado, escoimado dos conhecimentos que estão na sua gênese científicotecnológica e na sua apropriação histórico-social. Como formação humana, o que se busca é garantir ao adolescente, ao jovem e ao adulto trabalhador o direito a uma formação completa para a leitura do mundo e para a atuação como cidadão pertencente a um país, integrado dignamente à sua sociedade política. Formação que, neste sentido, supõe a compreensão das relações sociais subjacentes a todos os fenômenos.
\end{abstract}

Por formação humana integral, portanto, entende-se que a centralidade do processo educativo encontra-se no ser humano, enquanto sujeito pertencente a uma sociedade e ontologicamente ligado ao trabalho. Para que tal processo educativo se efetive, há a necessidade de se pensar na emancipação dos sujeitos. Emancipação, neste contexto, significa o ato de libertar os estudantes do caráter hegemônico presente nas relações de poder da sociedade a qual pertencem, dar a eles voz, de forma que possam, criticamente, conhecer e atuar no mundo em que vivem.

Discutir o tema 'emancipação humana' em suas relações com a educação, requer um entendimento sobre esta em seu sentido lato:

[...] um fenômeno humano e social, com suas determinações históricas. Educar é produzir o homem, construir sua identidade ontológica, social, cultural, étnica e produtiva. A educação é o campo da ação humana e, consequentemente, toda a sociedade ou qualquer grupo social é uma agência educadora. Não se reduz unilateralmente educação à escolarização ou instrução. [...]. Assim, a Educação é um conceito amplo que se refere ao processo de desenvolvimento omnilateral da personalidade, subjetiva e social, envolvendo a formação de qualidades humanas - físicas, morais, intelectuais, estéticas - tendo em vista a orientação da atividade humana na sua relação com o meio social, num determinado contexto de relações sociais (NUNES, 2001, p. 22).

Trata-se, portanto, do conceito de educação de acordo com a proposta de formação dos IFs, que prevê a formação omnilateral dos sujeitos, por meio de sua emancipação. A concepção de emancipação humana que se apresenta está articulada às proposições marxistas, já que, "no marxismo, autonomia individual e emancipação humana se complementam" (NUNES; FEITOZA, 2008). Não se trata, portanto, simplesmente da emancipação política ou do conceito restrito à cidadania, embora estas também façam parte da autonomia individual pretendida.

Na perspectiva da EPT, a ideia de emancipação humana é percebida como "a possibilidade de os seres humanos assumirem o controle da história de maneira consciente e planejada" (IASI, 2007 p. 67), o que só é possível por meio da real articulação entre teoria, reflexão e práxis. Contudo, Nunes enseja que:

[...] produzir autonomia crítica, cultural e simbólica, esclarecimento científico, libertação de toda forma de alienação e erro, de toda submissão, engodo, falácia ou pensamento colonizado, incapaz de esclarecer os processos materiais, culturais e políticos (NUNES, 2003, p. 35).

O evento apresentado, organizado pelo SIBi/IF Goiano em 2020, vai ao encontro das atividades inerentes à busca da emancipação humana plena, quando leva aos estudantes da instituição uma possibilidade de aprimoramento da competência em informação, tão necessária num contexto em que as redes digitais se tornam cada vez mais acessíveis e estas po- 
dem manipular seus usuários, de acordo com seus interesses, e em que as fake news trazem diversos danos às sociedades.

\subsection{AS BIBLIOTECAS DO INSTITUTO FEDERAL GOIANO}

Em 2014, o Sistema Integrado de Bibliotecas (SIBi) foi instituído por meio da Resolução CS no 010 de 21/02/2014, com a finalidade de proporcionar funcionamento integrado, otimizando a utilização e a gerência de acervos das bibliotecas no âmbito do IF Goiano. O SIBi é responsável pela definição de normas e diretrizes, as quais visam subsidiar as bibliotecas e demais unidades de informação na esfera do IF Goiano, na prestação de serviços e produtos de informação, de forma a atender as demandas de ensino, pesquisa e extensão. Ainda, tem a função de assessorar as bibliotecas dos campi, a fim de estabelecer condições e procedimentos para a utilização dos serviços, materiais e instalações, de forma a garantir o perfeito funcionamento de suas atividades e a qualidade do acervo e dos serviços oferecidos, com foco no atendimento aos usuários.

O SIBi/IF Goiano atualmente é composto por 12 (doze) bibliotecas, instaladas em seus respectivos campi. Enquanto missão, as bibliotecas buscam ser um espaço dinamizador, que possa colaborar com o ensino, sendo um local de descobertas, onde o aluno possa sentir-se inserido na instituição, tendo possibilidades de leituras diversas e de pesquisas que o auxiliem nesse processo. Por tratar-se de um suporte informacional e pedagógico, os professores podem trabalhar em conjunto com a biblioteca, para que os estudantes consigam usufruir plenamente das ferramentas que a mesma oferece.

No espaço físico das bibliotecas dos IFs não existe distinção para cada tipo de público, não há uma biblioteca destinada ao ensino médio técnico, e outra para o ensino superior e pós-graduação. O espaço é único, com o objetivo principal de atender a discentes, docentes, técnicos administrativos e comunidade externa, atuando em consonância com a missão da instituição na qual estão inseridas.

A literatura científica sobre as unidades de informação e bibliotecas dos IFs ainda divergem em relação à nomenclatura sobre sua tipologia, definindo-as com terminologias como mistas (BECKER; FAQUETI, 2015) ou multiníveis (MOUTINHO, 2014; ALMEIDA; FREIRE, 2018), por compreenderem que estas atendem aos usuários de diversos níveis de ensino (médio, técnico, graduação e pós-graduação), e por possuírem especificidades de diferentes tipos de bibliotecas em uma única estrutura, como demonstra o Quadro 1.

Quadro 1: Tipologia das bibliotecas dos Institutos Federais.

\begin{tabular}{|l|l|}
\hline $\begin{array}{l}\text { Biblioteca } \\
\text { escolar }\end{array}$ & $\begin{array}{l}\text { Os Institutos Federais podem ofertar cursos técnicos integrados ao ensino } \\
\text { médio. }\end{array}$ \\
\hline $\begin{array}{l}\text { Biblioteca } \\
\text { universitária }\end{array}$ & $\begin{array}{l}\text { Os Institutos Federais podem ofertar cursos superiores em tecnologia, licen- } \\
\text { ciaturas e Pós-Graduação Lato sensu e Stricto sensu. }\end{array}$ \\
\hline $\begin{array}{l}\text { Biblioteca } \\
\text { especializada }\end{array}$ & $\begin{array}{l}\text { Os Institutos Federais podem ofertar cursos específicos em uma área para } \\
\text { atender a demanda da comunidade }\end{array}$ \\
\hline
\end{tabular}

Fonte: elaborado pelos autores (2020).

Conforme apresentado no Quadro 1, as Bibliotecas dos IFs possuem múltiplas especificidades e diversos públicos dentre os usuários. Nesse sentido, atendem às várias funções de biblioteca escolar, universitária, especializada e, em alguns casos, pública. Assim, os pro- 
fissionais bibliotecários possuem o compromisso com a realização de ações educativas, que visem o papel pedagógico da biblioteca, além de aliar seus produtos e serviços ao ensino, pesquisa e extensão.

No contexto da formação integral e emancipação dos indivíduos, a biblioteca pode corroborar com os princípios da EPT. De acordo com Castrillõn (1985), o conceito de biblioteca parte da análise de suas funções com relação ao sistema educativo, ao currículo, à leitu$\mathrm{ra}$, ao desenvolvimento da capacidade de pesquisa, de criatividade, com a aprendizagem permanente, a comunicação, a recreação, a capacitação do professor, a informação educativa e a relação com a comunidade. Sob esse viés, a SNBL do SIBi/IF Goiano, em 2020, proporcionou um evento para discutir fake news e inteligência artificial, temas necessários para compreender as informações que são recebidas, principalmente nos ambientes digitais, os quais estão cada dia mais inseridos na sociedade.

\subsection{SEMANA NACIONAL DO LIVRO E DA BIBLIOTECA}

A Semana Nacional do Livro e da Biblioteca é um evento comemorativo, instituído pelo Decreto no 84.631, de 09 de abril de 1980, com data demarcada no período de 23 a 29 de outubro (BRASIL, 1980). No ano de 2020, o SIBi/IF Goiano optou por comemorar a SNBL entre os dias 13 a 16 de outubro, devido aos esforços necessários durante a pandemia da Covid-19 e, também, para conciliar com a Semana Nacional de Ciência e Tecnologia, promovida pelo IF Goiano, dentre outros fatores, como logística e disponibilidade dos profissionais participantes. Esse ano, excepcionalmente, o evento ocorreu de forma virtual, por questões sanitárias exigidas pela conjuntura da pandemia.

O evento contou com a participação de vários profissionais para sua realização, toda a equipe de bibliotecários e auxiliares das bibliotecas do IF Goiano, além de parcerias elaboradas com docentes, técnicos administrativos, estudantes e egressos da instituição. Becker e Faquetti (2015) salientam que a profissão de bibliotecário está arraigada por conceitos técnicos, que pouco expõem sua função social e educativa, no sentido de auxiliar a comunidade de usuários na utilização das fontes de informação, de ensinar o usuário a buscar, analisar e compreender a informação, de incentivar o estudante ou pesquisador a ler e de oportunizar momentos culturais.

Nessa perspectiva, o projeto da SNBL do SIBi/IF Goiano busca contribuir para a função educativa das bibliotecas, oportunizando aos seus usuários a participação em ações que corroborem com a formação crítica e reflexiva, visando à autonomia e emancipação dos estudantes.

Conforme assinala Lubisco (2011), é importante que a biblioteca se integre à instituição a qual pertence, por meio de um planejamento participativo que contribua com a missão institucional, e que o olhar sobre as bibliotecas seja como um recurso pedagógico indispensável para as atividades nos três níveis: ensino, pesquisa e extensão.

\subsubsection{Fake News e Inteligência Artificial}

A inteligência artificial (IA), como disciplina do conhecimento humano, vem se desenvolvendo constantemente. Suas capacidades se assemelham cada vez mais com as dos seres humanos, desde a tomada de decisões, reconhecimento visual, reconhecimento de voz, traduções, entre outros (KAUFMAN, 2020). O uso da IA não se restringe a problemas específicos. Devido à generalização de suas capacidades, a sua popularidade aumentou drastica- 
mente no decorrer dos anos. Porém, ao mesmo tempo em que é uma ferramenta muito poderosa para promover a solução de problemas considerados como legítimos, pode também ser utilizada para criação de malefícios para a sociedade. Um exemplo de prejuízo que a IA foi capaz de auxiliar na promoção, foram as fake news.

O conceito de fake news não foi criado recentemente. Entretanto, ficou muito famoso a partir da segunda década do século XXI, devido à grande quantidade de notícias falsas tradução literal do termo - criadas e disseminadas nos meios de comunicação digital. As fake news são informações passadas ao público sem fundamentação ou veracidade, com o objetivo de enganar o leitor (LIM JUNIOR; LING, 2017).

Dentre as várias técnicas de IA que podem ser utilizadas na elaboração de fake news, está a Rede Adversária Generativa (do inglês Generative Adversarial Network), popularmente conhecida como Deep Fake. Com o uso dessa ferramenta, é possível manipular imagens de pessoas, desde a troca de rostos até a criação de pessoas inéditas, ou seja, que nunca nasceram (DOLHANSKY et al., 2020).

Na Figura 1, exemplifica-se o resultado da utilização dessa técnica, para demonstrar o quanto é difícil identificar quando uma imagem é verdadeira ou falsa, pois é imperceptível a diferença entre uma imagem criada e uma realista, podendo confundir ou enganar as pessoas quanto à veracidade da mesma.

Figura 1: Comparação entre uma foto real de Barack Obama com a gerada pela Deep Fake

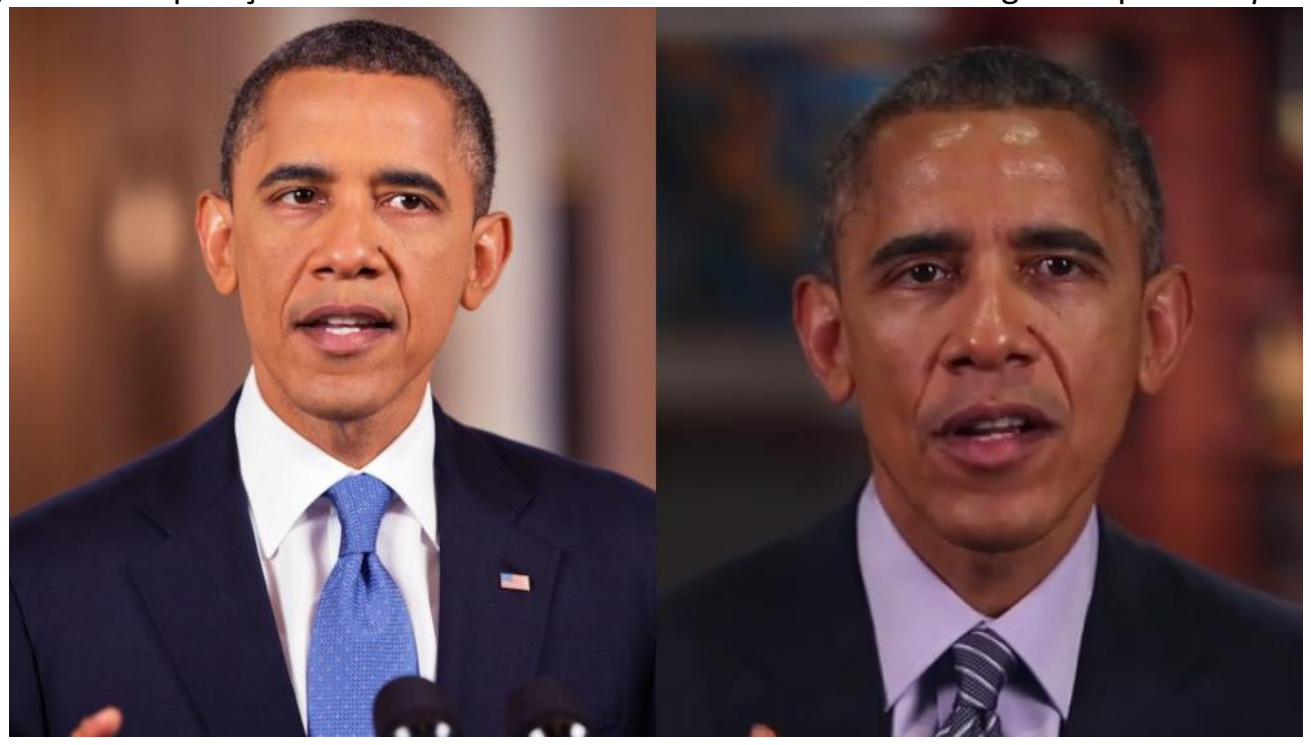

Fonte: (GIRLA, 2017) e (SOARES, 2018).

$O$ advento da IA proporcionou a obtenção de resultados positivos e negativos para a humanidade (KAUFMAN; SANTAELLA, 2020). Contudo, assim como diversas outras tecnologias e ferramentas, é de extrema necessidade saber utilizar e aproveitar os seus benefícios em detrimento aos malefícios promovidos. Sob esse viés, torna-se necessário o debate acerca do tema, para que possam ser sanadas dúvidas e obter conhecimentos sobre essas tecnologias.

Foi nessa perspectiva que o evento da SNBL do SIBi/IF Goiano, em 2020, procurou levantar discussões sobre IA, como estas podem contribuir para a elaboração de fake news e seu impacto na sociedade. 


\section{RESULTADOS E DISCUSSÕES}

Várias ações culturais foram programadas para o evento, que se encontra na sexta edição. Neste trabalho, ressalta-se a roda de conversa realizada em uma live, na plataforma do Youtube, a qual foi apresentada por duas bibliotecárias e um docente da área de Ciência da Computação, para explicitar sobre fake news e inteligência artificial para a comunidade acadêmica do IF Goiano.

A divulgação do evento foi realizada por meio de mídias sociais em plataformas virtuais, tais como Instagram, Facebook, WhatsApp, além de e-mails e publicações nos sites oficiais da instituição. Com o intuito de aliar o conteúdo ao contexto pandêmico atual, e também à Semana Nacional da Ciência e Tecnologia, realizada pelo IF Goiano, com o tema "Inteligência artificial: a nova fronteira da ciência brasileira", o assunto proposto pela SNLB do SIBi/IF Goiano foi: "Fake news - o lado incógnito da Inteligência Artificial".

A programação da SNBL do SIBi/ IF Goiano foi elaborada por meio de estudos e reuniões virtuais com a equipe de bibliotecários. O propósito foi discutir um tema atual e presente na sociedade, que afetasse a tomada de decisão dos indivíduos, interferindo em suas vidas. Para tal finalidade, tentou-se desvelar as estratégias dos sistemas digitais, para que os estudantes, de posse deste conhecimento, pudessem utilizar o senso crítico para interpretarem as informações que recebem.

A live obteve uma participação considerável, apesar de terem ocorrido problemas técnicos no início. Durante a transmissão, houveram 64 acessos simultâneos e, posteriormente, o vídeo gravado já conta com mais de 290 visualizações (até março/2021), no canal do SIBi/ IF Goiano no Youtube. Os comentários instantâneos proporcionaram um debate construtivo sobre o tema, vários participantes discorreram sobre o pouco conhecimento que dispunham sobre o assunto e o quanto é importante sua discussão. Várias questões foram levantadas pelos participantes e esclarecidas pelos profissionais convidados. Muitos estudantes manifestaram surpresa na apresentação dos sistemas de IA e relataram que desconheciam tais processos.

A avaliação do momento foi classificada como positiva pelos organizadores do evento, devido à quantidade de pessoas presentes na live, bem como ao proveitoso debate suscitado e à elucidação dos conteúdos que compõem o tema proposto. Esses esclarecimentos são bastante necessários para que os estudantes possam ampliar seus conhecimentos, compreendendo que as informações que recebem podem ser manipuladas de acordo com os interesses de quem as disseminam, sendo capazes de ter autonomia e uma visão crítica de mundo para entender as contradições da sociedade globalizada.

Visando combater o fenômeno das fake news, diversas iniciativas podem ser desenvolvidas por meio de instituições governamentais, educacionais, organismos de classes, discussões independentes entre estudiosos da temática, dentre outras. As bibliotecas, por meio dos serviços e produtos desenvolvidos pelos bibliotecários, podem auxiliar os indivíduos no melhor uso das informações, evitando que os mesmos contribuam com a propagação de fake news. A exemplo, a live objeto deste trabalho, uma iniciativa de bibliotecários de uma instituição educacional.

O uso ético no contexto informacional enquadra-se em uma das dimensões da competência em informação. Para Vitorino e Piantola (2011), o exercício do comportamento ético quanto ao uso da informação diz respeito a utilização responsável de informações, aspirando o bem estar coletivo. Tendo em vista que o compartilhamento de informações falsas 
vai de encontro ao uso consciente das mesmas, a competência em informação dá subsídios para que os sujeitos possam se orientar por uma conduta correta e ética, quando envolvidos com o universo informacional, sabendo como avaliar suas fontes para o uso eficiente. Desse modo, ressalta-se que a iniciativa aqui relatada pode ser considerada meio para desenvolver competência em informação para os discentes e comunidade em geral.

\section{CONSIDERAÇÕES FINAIS}

Considera-se que as bibliotecas, nos sistemas educacionais da EPT, podem contribuir para a formação do estudante e suas lides com a informação. No viés da formação humana integral dos sujeitos, estas se tornam uma ferramenta essencial para a promoção da emancipação dos mesmos, colaborando com seu protagonismo na sociedade.

Espera-se que ações como a Semana Nacional do Livro e da Biblioteca possam ser cada vez mais recorrentes nos espaços virtuais, para que possam alcançar uma maior quantidade de pessoas, mesmo em um contexto pandêmico, em que o distanciamento social é imposto por questões sanitárias. Entende-se que projetos como o relatado contribuem para a discussão de temas relevantes para a sociedade e suscitam questionamentos e críticas em torno dos mesmos.

Compreende-se que futuras pesquisas com foco nas interrelações entre as fake news e competência em informação, tendo como sujeitos os profissionais bibliotecários, bem como pesquisas com os discentes da EPT e suas percepções sobre o papel das bibliotecas no combate às fake news, poderiam corroborar para um constructo de elementos que fomentam tais práticas para o desenvolvimento de cidadãos críticos e reflexivos perante o contexto informacional e a sociedade.

\section{REFERÊNCIAS}

ALMEIDA, J. L. S.; FREIRE, G. H. A. A biblioteca multinível no IFPB campus Sousa: conceito, descrição e finalidade. Informação \& Informação, Londrina, v. 23, n. 2, p. 520 - 537, maio/ago. 2018. Disponível em: <http://srv009.uel.br/revistas/uel/index.php/informacao/article/view/31017/24215>. Acesso em: 20 out. 2020.

ALMEIDA, J. L. S.; FREIRE, G. H. A. Projeto educativo para competências em informação: bases teóricas para a pesquisa-ação em uma biblioteca multinível. Encontros Bibli: Revista Eletrônica de Biblioteconomia e Ciência da Informação, v. 23, n. 51, p. 123-137, 2018. Disponível em: <http://www.brapci.inf.br/index.php/res/v/39117>. Acesso em: 19 out. 2020.

BECKER, C. R. F.; FAQUETI, M. F. Panorama das bibliotecas da Rede Federal de Educação Profissional, Científica e Tecnológica: um olhar sobre a gestão. Blumenau: IFC, 2015. Disponível em: <http://editora.ifc.edu.br/wpcontent/uploads/sites/33/2017/06/Panorama-dasbibliotecas-da-Rede-Federal-deEduca\%C3\%A7\%C3\%A3o-Profissional-Cient\%C3\%Adfica-eTecnol\%C3\%B3gica-umolhar-sobre-a-gest\%C3\%A3o.pdf>. Acesso em: 11 out. 2020.

BRASIL. Decreto no 84.631, de 09 de abril de 1980. Institui a Semana Nacional do Livro e da Biblioteca e o Dia do Bibliotecário. Diário Oficial da União: seção 1, Brasília, DF, p. 6491, 15 abr. 1980. Disponível em: 
<http://www.planalto.gov.br/ccivil_03/Atos/decretos/1980/D84631.html>. Acesso em: 20 out. 2020.

CASTRILLÓN, S. Modelo flexível para um sistema nacional de bibliotecas escolares. Brasília: FEBAB, 1985.

CIAVATTA, M. A formação integrada: a escola e o trabalho como lugares de memória e de identidade. In: FRIGOTTO, Gaudêncio; CIAVATTA, Maria; RAMOS, Marise (org.). Ensino médio integrado: concepção e contradições. São Paulo: Cortez, 2005, p. 83-105.

DOLHANSKY, B. et al. The DeepFake Detection Challenge (DFDC) Dataset. Cornell University, Nova York, out. 2020. Disponível em: <https://arxiv.org/abs/2006.07397>. Acesso em: 07 out. 2020.

GIRLA, E. Obama would not be selected even as President of Moldova, based on his Linkedln profile. Medium. 2017. Disponível em: <https://medium.com/gaus/obama-would-not-beselected-even-as-president-of-moldova-based-on-his-linkedin-profile-c4b861a7383>. Acesso em: 18 mar. 2021.

IASI, M. L. Ensaios sobre consciência e emancipação. São Paulo: Expressão Popular, 2007.

INSTITUTO FEDERAL DE EDUCAÇÃO, CIÊNCIA E TECNOLOGIA GOIANO. Conselho Superior. Resolução no 10, de 21 de fevereiro de 2014. Dispõe sobre a criação do Sistema Integrado de Bibliotecas. Goiânia: Conselho Superior, 2014. Disponível em:

$<$ https://www.ifgoiano.edu.br/home/images/RV/Biblioteca/Regulamento-SistemaIntegrado-de-Bibliotecas-SIBI_4_1.pdf>. Acesso em: 15 out. 2020.

KAUFMAN, D. Inteligência artificial: repensando a mediação. Brazilian Journal of Development, Curitiba, v. 6, n. 9, p. 66742-66760, set. 2020. Disponível em: <https://www.brazilianjournals.com/index.php/BRJD/article/view/16481>. Acesso em: 17 out. 2020.

KAUFMAN, D.; SANTAELLA, L. O papel dos algoritmos de inteligência artificial nas redes sociais. Revista FAMECOS, Porto Alegre, v. 27, p. 1-10, jan/dez. 2020. Disponível em: <https://revistaseletronicas.pucrs.br/index.php/revistafamecos/article/view/34074/19629>. Acesso em: 21 mar. 2021.

LIM JUNIOR, E. C. T.; Zheng W.; LING, R. Defining "Fake News": A typology of scholarly definitions. Digital Journalism, [S. I.], v. 6, n. 2, p. 137-153, ago. 2017. Disponível em: $<$ https://edisciplinas.usp.br/pluginfile.php/4948550/mod_resource/content/1/Fake\%20New s\%20Digital\%20Journalism\%20-\%20Tandoc.pdf>. Acesso em: 19 out. 2020.

LUBISCO, N. M. L. (org.). Biblioteca universitária: elementos para o planejamento, avaliação e gestão. Salvador: EDUFBA, 2011. Disponível em: <https://repositorio.ufba.br/ri/bitstream/ri/5620/1/_Biblioteca.pdf>. Acesso em: 07 out. 2020. 
MATIAS-PEREIRA, J. Manual de metodologia da pesquisa científica. 4. ed. rev. São Paulo: Atlas, 2016.

MOURA, D. H. Educação básica e educação profissional e tecnológica: dualidade histórica e perspectivas de integração. Holos, Natal, ano 23, v. 2, p. 04-30, 2007. Disponível em: <http://www2.ifrn.edu.br/ojs/index.php/HOLOS/article/view/11>. Acesso em: 19 out. 2020.

MOUTINHO, S. O. M. Práticas de leitura na cultura digital de alunos do ensino técnico integrado do IFPI - Campus Teresina Zona Sul. 2014. 183 f. Dissertação (Mestrado em Educação) - Universidade Vale do Rio dos Sinos, São Leopoldo, 2014. Disponível em: <http://www.repositorio.jesuita.org.br/handle/UNISINOS/3075>. Acesso em: 20 out. 2020.

NUNES, C. Educar para a emancipação. Florianópolis: Sophos, 2003.

NUNES, C. Filosofia, educação e emancipação: fundamentos éticos para uma prática política transformadora na sociedade brasileira do século XXI. Revista de Educação, São Paulo, v. 4, n. 04, nov. 2001.

NUNES, C.; FEITOZA, R. Os movimentos sociais e as políticas educacionais diante da questão da emancipação humana: as tendências reais e as novas ilusões repostas. Quaestio - Revista de Estudos em Educação, Sorocaba, SP, v. 10, n.1/2, p. 71-94, maio/nov. 2008. Disponível em: <http://periodicos.uniso.br/ojs/index.php/quaestio/article/view/62>. Acesso em: 23 mai. 2021.

PEREIRA, A. S.; SHITSUKA, D. M.; PARREIRA, F. J.; SHITSUKA, R. Metodologia da pesquisa científica. Santa Maria: UFSM. Disponível em: $<$ https://www.ufsm.br/app/uploads/sites/358/2019/02/Metodologia-da-PesquisaCientifica_final.pdf $>$. Acesso em: 18 mar. 2021.

SOARES, C. Deepfake: Você não vai acreditar no que Barack Obama disse neste vídeo. Medium. 2018. Disponível em: <https://medium.com/@cssoaresonline/deepfake-voc\%C3\%AAn\%C3\%A3o-vai-acreditar-no-que-barack-obama-diz-neste-v\%C3\%ADdeo-3892287b375a>. Acesso em: 18 mar. 2021.

VITORINO, E. V.; PIANTOLA, D. Dimensões da competência informacional. Ciência da Informação, Brasília, DF, v. 40, n. p. 99-110, jan./abr. 2011. Disponível em: <https://www.scielo.br/pdf/ci/v40n1/a08v40n1.pdf>. Acesso em: 18 mar. 2021. 